\title{
A Simple Explanation of the Information Paradox by the Information Model of a Black Hole
}

\section{Igor Gurevich}

The Institute of Informatics Problems of the Russian Academy of Sciences, Hetnet Consulting Corporation, Moscow, Russia

Email: iggurevich@gmail.com

Received 30 June 2014; revised 30 July 2014; accepted 9 August 2014

Copyright (C) 2014 by author and Scientific Research Publishing Inc.

This work is licensed under the Creative Commons Attribution International License (CC BY). http://creativecommons.org/licenses/by/4.0/

(c) (i)

\section{Abstract}

The information paradox first surfaced in the early 1970s when Stephen Hawking of Cambridge University suggested that black holes are not totally black. Hawking showed that particle-antiparticle pairs generated at the event horizon-the outer periphery of a black hole-would be separated. One particle would fall into the black hole while the other would escape, making the black hole a radiating body. Characteristics of the emission and absorption of usual substance by a black hole can be described by information models. Estimation of the volume of information in black holes is necessary for generation of restrictions for their formation, development and interconversion. Information is an integral part of the Universe. By its physical essence information is heterogeneity of matter and energy. Therefore information is inseparably connected with matter and energy. An information approach along with a physical one allows to obtain new, sometimes more general data in relation to data obtained on the ground of physical rules only. The author's works, testify about the practicality of information laws usage simultaneously with physical rules for cognition of the Universe. The results presented in this paper show the effectiveness of informational approach for studying the black holes. The article discusses the following questions: The volume of information in the black hole; Information model of a black hole; Characteristics of the emission and absorption of usual substance by a black hole describes the information model of a black hole; The information paradox; A simple explanation of the information paradox by the information model of a black hole.

\section{Keywords}

Black Hole, Usual Substance, Information, Models, Volume, Universe, Paradox 


\section{The Information Paradox}

The information paradox first surfaced in the early 1970s when Stephen Hawking of Cambridge University, building on earlier work by Jacob Bekenstein at the Hebrew University of Jerusalem, suggested that black holes are not totally black. Hawking showed that particle-antiparticle pairs generated at the event horizon-the outer periphery of a black hole-would be separated. One particle would fall into the black hole while the other would escape, making the black hole a radiating body. Hawking's theory implied that, over time, a black hole would eventually evaporate away, leaving nothing. This presented a problem for quantum mechanics, which dictates that nothing, including information, can ever be lost. If black holes withheld information forever in their singularities, there would be a fundamental flaw with quantum mechanics.

The significance of the information paradox came to a head in 1997 when Hawking, together with Kip Thorne of the California Institute of Technology (Caltech) in the US, placed a bet with John Preskill, also of Caltech. At the time, Hawking and Thorne both believed that information was lost in black holes, while Preskill thought that it was impossible. Later, however, Hawking conceded the bet, saying he believed that information is returnedalbeit in a disguised state. At the turn of this century, Maulik Parikh of the University of Utrecht in the Netherlands, together with Frank Wilczek of the Institute of Advanced Study in Princeton, US, showed how information could leak away from a black hole. In their theory, information-carrying particles just within the event horizon could tunnel through the barrier, following the principles of quantum mechanics. But this solution, too, remained debatable [1]-[5]. Information paradox is easily explained with the use of the information model of a black hole.

\section{The Volume of Information in the Black Hole}

The basic law of Zeilinger's quantum mechanics postulates that the elementary physical system (in particular, fundamental particles: quark, electron, photon) bears one bit of information [6].The volume of information in physical systems is estimated the information entropy [7]-[10]. The information volume of a black hole of mass $M_{B h}$ is equal to $I_{B h}=\frac{2 \pi \cdot G}{\hbar \cdot C} M_{B h}^{2}$ nat, or $I_{B h}=\frac{2 \pi \cdot G}{\hbar \cdot c \cdot \ln 2} M_{B h}^{2}$ bit [11]-[13], where $\hbar$ is the reduced Planck constant, $c$ is the speed of light, $k_{\mathrm{B}}$ is the Boltzmann constant, $G$ is the gravitational constant, and $M_{B h}$ is the mass of the black hole.

The information volume contained in the black hole is proportional to its squared mass. How to explain it? Let us assume that a black hole contains $n$ pairwise interacting particles (q-bits). Then the quadratic dependence of the volume of information in the black hole on its mass can be explained by the fact that each interaction forms 1 bit of information. The black hole is described with the wave function

$\psi_{n}=\frac{1}{\sqrt{2}}\left(\left|0_{1}\right\rangle\left|0_{2}\right\rangle \cdots\left|0_{n}\right\rangle+\left|1_{1}\right\rangle\left|1_{2}\right\rangle \cdots\left|1_{n}\right\rangle\right)$. As mentioned earlier the volume of information in the system described with the present given function is equal to $I_{n}=\frac{n \cdot(n+1)}{2}$ bit. At $n \gg 1$ the volume of information in the black hole is proportional to the black hole squared mass $I_{n} \approx \frac{n^{2}}{2}$. A black hole consisting of $n$ black particles contains the information, which is $n$ bit larger than a black hole consisting of $n-1$ particles: $I_{n}-I_{n-1}=\frac{n(n+1)}{2}-\frac{(n-1) \cdot n}{2}=n$.

Let us compare the estimates of squared mass of a black hole: $M_{B h}^{2}=\frac{\hbar \cdot c \cdot \ln 2}{2 \pi \cdot G} \cdot I_{B h}$ (from cosmological formula [11]-[13]) and $M_{B h}^{2}=n^{2} \cdot m_{0}^{2}$ (squared mass $n$ of the particles composing the black hole). We have $M_{B h}^{2}=n^{2} \cdot m_{0}^{2}=\frac{\hbar \cdot c \cdot \ln 2}{2 \pi \cdot G} \cdot I_{B h}$. As far as $I_{u d}=\frac{n^{2}}{2}$, then $n^{2} \cdot m_{0}^{2}=\frac{\hbar \cdot c \cdot \ln 2}{2 \pi \cdot G} \cdot \frac{n^{2}}{2}$, or $m_{0}^{2}=\frac{1}{4 \pi} \cdot \frac{\hbar \cdot c \cdot \ln 2}{G}$. Eventually we obtain the estimate of mass of particles being part of the black holes: $m_{0}=\frac{\sqrt{\ln 2}}{2 \sqrt{\pi}} \sqrt{\frac{\hbar \cdot c}{G}}=\frac{\sqrt{\ln 2}}{2 \sqrt{\pi}} \sqrt{\frac{h \cdot c}{2 \pi \cdot G}} \mathrm{~g}$, that agree by its magnitude with Planck mass 
$m_{P l}=\sqrt{\frac{\hbar \cdot c}{G}} \approx 2.17 \times 10^{-5} \mathrm{~g}: m_{0}=\frac{\sqrt{\ln 2}}{2 \sqrt{\pi}} m_{P l} \approx 0,23 \sqrt{\frac{\hbar \cdot c}{G}}=5.09 \times 10^{-6} \mathrm{~g}$, where $m_{P l}$ is the mass of the Planck particle, $m_{0}$ mass of the particles composing the black hole.

Therefore, a black hole is the aggregate of particles (let us call them black particles) each having a mass equal to 0.23th of Planck mass) and interacting with all other black particles that form a black hole. Characteristics and models used in the paper are taken from [14]-[22].

\section{Emission and Absorption of Usual Substance by a Black Hole}

Suppose that at the initial instant of time a black hole consisting of $n$ black particles has the mass of $M_{n}=n \cdot m_{0}$ and contains $I_{n}=\frac{n \cdot(n+1)}{2}$ bit of information. The black hole mass changes (increases or decreases) by black particles (quanta) $\Delta M=m_{0}$. As far as each black hole has the sub-Planck mass $m_{0}$, then the change in energy of a black hole in such a case is equal to $\Delta E=m_{0} c^{2}$. In the event of the loss of emission of one black particle the mass of a black hole becomes equal to $M_{n}=(n-1) \cdot m_{0}$. In this case the volume of information remaining in the black hole is $I_{n-1}=\frac{(n-1) \cdot n}{2}$ bit. Change (loss) of information in the black hole comes to $n$ bit.

For further estimates we implement the law of conservation of uncertainty (information) [14]-[23] and the law of conservation energy. According to the law of conservation of uncertainty (information) a change in the system "a black hole with the mass $M_{n}=n \cdot m_{0}$ - external environment" on emission of one black particle must be balanced by the occurrence of $n$ particles containing 1 bit each: $I_{n}-I_{n-1}=n$ bit. It can believed that $n$ photons of frequency $v$ and energy $h v$ emerged.

In virtue of energy conservation principle $n \cdot h v=m_{0} c^{2}$ and $v=\frac{m_{0} c^{2}}{n \cdot h}$ Because $m_{0}=\frac{\sqrt{\ln 2}}{2 \sqrt{\pi}} \sqrt{\frac{\hbar \cdot c}{G}}$, then the frequency of emitted photon must be equal to $v=\frac{1}{n} \cdot \frac{\sqrt{\ln 2}}{2 \sqrt{\pi}} \sqrt{\frac{c^{5}}{h \cdot G}}=\frac{4.34 \times 10^{42}}{n} 1 / \mathrm{c}$. Because $t_{P l}=\sqrt{\frac{\hbar \cdot G}{c^{5}}} \approx 5.41 \times 10^{-44} \mathrm{c}$, then $v=\frac{1}{n} \cdot \frac{\sqrt{\ln 2}}{2 \sqrt{\pi} \cdot t_{P l}}$. Let us denote $v_{0}=\frac{\sqrt{\ln 2}}{2 \sqrt{\pi}} \frac{1}{t_{P l}}=\frac{\sqrt{\ln 2}}{2 \sqrt{\pi}} \sqrt{\frac{c^{5}}{\hbar \cdot G}} \approx 0.43 \times 10^{43} 1 / \mathrm{c}$. Then $v=\frac{v_{0}}{n}$.

In the case of a black hole containing one black particle the radiation frequency is maximal and in inverse proportion to Planck time unit. Similar dependences are true for absorption of photons by black holes.

In the general case, there must appear $n$ photons having the frequency of $v_{i}$ and aggregated energy $h \sum_{i=1}^{n} v_{i}=m_{0} c^{2}$ equal to $n$ bit.

In the general case, there must appear $k$ particles with aggregated information equal to $n$ bit and aggregated energy $m_{0} c^{2}$.

In the general case, there must be absorbed $n$ photons having the frequencies $v_{i}$ and the aggregated energy $h \sum_{i=1}^{n} v_{i}=m_{0} c^{2}$ equal to $n$ bit.

In the general case, there must be absorbed $k$ particles with aggregated information equal to $n$ bit and aggregated energy $m_{0} c^{2}$.

Identical dependencies are true for cases when photons are absorbed by black holes. In virtue of the law of conservation of uncertainty (information), the changes in the system "a black hole with the mass $M_{n-1}=(n-1) \cdot m_{0}$ - external environment”, when the mass of a black hole is increased by the mass of one black particle, must be determined by absorption of $n$ particles containing 1 bit each: $I_{n}-I_{n-1}=n$ bit. Suppose that there had been absorbed $n$ photons of frequency $v$ and energy $h v$. By virtue of energy conservation 
principle, $n \cdot h v=m_{0} c^{2}$. The frequency of each absorbed photon must be equal to $v=\frac{1}{n} \cdot \frac{\sqrt{\ln 2}}{2 \sqrt{\pi}} \sqrt{\frac{c^{5}}{\hbar \cdot G}}=\frac{4.34 \times 10^{42}}{n} 1 / \mathrm{sec}$. At the formation of a black hole consisting one black particle the absorption frequency is maximal and inversely proportional to Planck time unit.

Having the estimates of black holes distribution by mass one can calculate the intensity of aggregated distribution of black holes radiation by frequencies and compare them with the experiment results. From the obtained radiation frequency expression one can draw the estimate of black hole radiation temperature. We calculate the temperature of the radiation of a black hole. The thermal radiation of a black body is related to the average energy of radiation $h v=2.7 k T$, or $T=\frac{h v}{2.7 k}$. Since $v=\frac{m_{0}^{2} c^{2}}{M \cdot h}=\frac{\ln 2}{8 \pi^{2}} \frac{c^{3}}{M \cdot G}$, then $T=\frac{h v}{2.7 k}=\frac{h}{2.7 k} \frac{\ln 2}{8 \pi^{2}} \frac{c^{3}}{M \cdot G}=\frac{\ln 2 \cdot \hbar \cdot c^{3}}{4 \pi \cdot 2.7 \cdot k \cdot M \cdot G}$. The resulting estimate of temperature radiation from the black hole mass can be called information estimate of temperature, which coincides up to a factor $\frac{2 \ln 2}{2.7} \approx 0,51$ with an estimate of Hawking $T_{H}=\frac{\hbar \cdot c^{3}}{8 \pi \cdot k \cdot G \cdot M}$ [23].

\section{Conclusions}

1) The volume of information in the black hole is proportional to the square of the mass black hole.

2) The information model of a black hole is:

- A black hole is the aggregate of $n$ particles (black particles, q-bits) each having a mass equal to 0.23th of Planck mass) and interacting with all other black particles that form a black hole.

- The black hole is described with the wave function

$$
\psi_{n}=\frac{1}{\sqrt{2}}\left(\left|0_{1}\right\rangle\left|0_{2}\right\rangle \cdots\left|0_{n}\right\rangle+\left|1_{1}\right\rangle\left|1_{2}\right\rangle \cdots\left|1_{n}\right\rangle\right) .
$$

- Volume of information in the system described with the present wave function is equal to $I_{n}=\frac{n \cdot(n+1)}{2}$ bit. At $n \gg 1$ the volume of information in the black hole is proportional to the black hole squared mass $I_{n} \approx \frac{n^{2}}{2}$.

3) The temperature of the radiation of a black hole coincides up to a factor $\frac{2 \ln 2}{2.7} \approx 0.51$ with an estimate of Hawking radiation $T_{H}=\frac{\hbar \cdot c^{3}}{8 \pi \cdot k \cdot G \cdot M}$.

4) A simple explanation of the information paradox with the information model of a black hole and the laws of conservation of uncertainty (information) and of conservation energy was made.

\section{Acknowledgements}

The author thanks N. Kardashev, I. Novikov, I. Sokolov, S. Shorgin, V. Sinitsin, V. Lipunov, L. Gindilis, M. Abubekerov, and especially A. Panov for the shown interest and support of this direction, and also for useful discussions of stated ideas.

\section{References}

[1] Hawking, S.W. and Penrose, R. (1970) The Singularities of Gravitational Collapse and Cosmology. Proceedings of the Royal Society A: Mathematical, Physical and Engineering Sciences, 314, 529-548. http://dx.doi.org/10.1098/rspa.1970.0021

[2] Hawking, S. (1971) Gravitational Radiation from Colliding Black Holes. Physical Review Letters, 26, 1344-1346. 
http://dx.doi.org/10.1103/PhysRevLett.26.1344

[3] Hawking, S. (2008) The Universe in a Nutshell. Bantam Dell, New York. (Susskind Quashes Hawking in Quarrel Over Quantum Quandary, California Literacy Review)

[4] J. Cartwright (2011) Information Paradox Simplified. Physics World—The Member Magazine of the Institute of Physics. http://physicsworld.com/cws/article/news/2011/aug/15/information-paradox-simplified

[5] Black Hole Information Paradox. http://en.wikipedia.org/wiki/Black_hole_information_paradox

[6] Zeilinger, A. (1999) A Foundational Principle for Quantum Mechanics. Foundations of Physics, 29, 631-643. http://dx.doi.org/10.1023/A:1018820410908

[7] Shannon, C.E. (1948) A Mathematical Theory of Communication. Bell System Technical Journal, 27, 379-423. http://dx.doi.org/10.1002/j.1538-7305.1948.tb01338.x

[8] Stratonovich, R.L. (1975) Information Theory. Soviet Radio, Moscow. (in Russian)

[9] Brillouin, L. (1960) Science and Information Theory. Fizmatgiz, Moscow, 392 p. (in Russian)

[10] Valiev, K.A. and Kokin, A.A. (2004) Quantum Computers: Hope and Reality. Scientific and Publishing Center "Regular and Chaotic Dynamics”, Moscow-Izhevsk, 320. (in Russian)

[11] Penrose, R. (1989) The Emperor’s New Mind. Oxford University Press, Oxford, 466 p.

[12] Novicov, I.D. and Frolov, V.P. (1986) The Pfysics of Black Holes. Science, Moscow, 328 p. (in Russian)

[13] Vasiliev, A.N. (1996) Evolution of the Universe. The St. Petersburg State University. (in Russian) http://www.astronet.ru:8101/db/msg/1210286

[14] Gurevich, I.M. (1989) Law of Informatics-A Basis of Researches and Designing of Complex Communication and Management Systems. Ecos, Moscow, 60 p. (in Russian)

[15] Gurevich, I.M. (2007) Informatics Laws-A Basis of a Structure and Cognitive of Complex Systems. 2nd Edition, Torus Press, Moscow. (in Russian)

[16] Gurevich, I.M. (2007) Information Model of a Black Hole. Proceedings of the Conference VAK-2007, Kazan, 2007, 461-462.

[17] Gurevich, I.M. (2007) Of Information Models in Cosmology. Systems and Tools of Computer Science. Vol. 17, IPI RAN, Moscow, 164-183.

[18] Gurevich, I.M. (2009) Information Characteristics of Physical Systems. “The 11th FORMAT”, Cypress, Moscow, Sevastopol, $170 \mathrm{p}$.

[19] Gurevich, I.M. (2010) Information Characteristics of Physical Systems. 2nd Edition Refined and Updated. Cypress, Sevastopol, $260 \mathrm{p}$.

[20] Gurevich, I.M. and Ursul, A.D. (2011) Information-The General Property of Matter. The Characteristics. The Estimations. The Restrictions. The Consequences. LIBROKOM, $312 \mathrm{p}$.

[21] Gurevich, I. (2012) Physical Informatics. LAP Lambert Academic Publishing GmbH \& Co. KG., Saarbrücken, 288 p.

[22] Gurevich, I. (2012) Some Works on Physical Informatics. LAP Lambert Academic Publishing, Saarbrücken, 276 p.

[23] Hawking Radiation. http://en.wikipedia.org/wiki/Hawking_radiation 
Scientific Research Publishing (SCIRP) is one of the largest Open Access journal publishers. It is currently publishing more than 200 open access, online, peer-reviewed journals covering a wide range of academic disciplines. SCIRP serves the worldwide academic communities and contributes to the progress and application of science with its publication.

Other selected journals from SCIRP are listed as below. Submit your manuscript to us via either submit@scirp.org or Online Submission Portal.
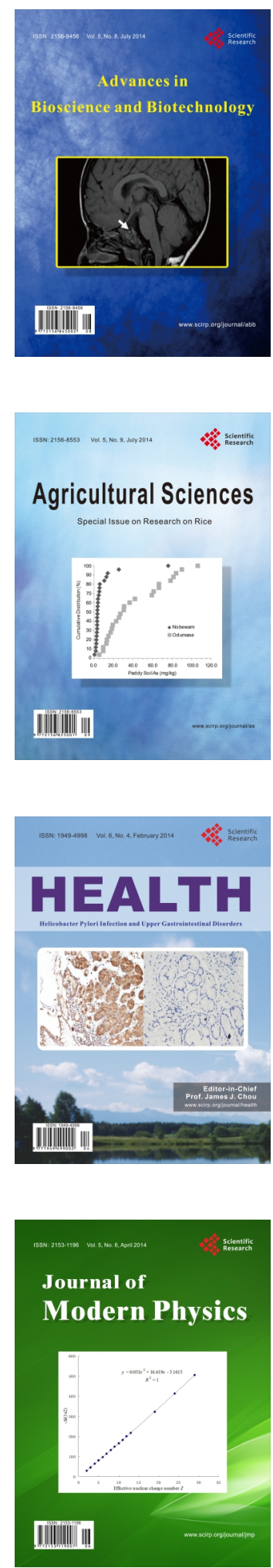
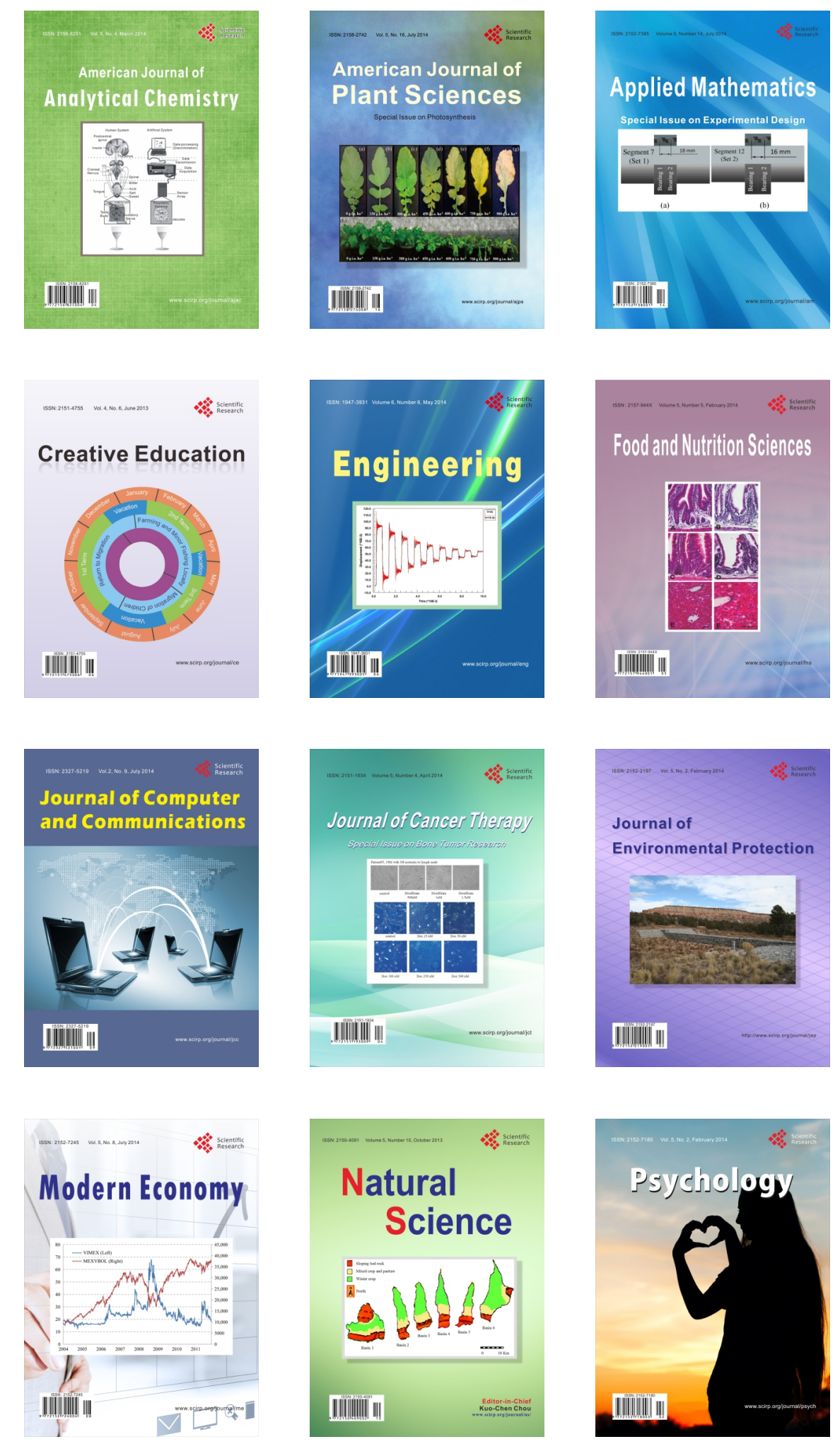